www.nature.com/clinicalpractice/uro

lobe or markedly enlarged prostate gland; and no tissue is obtained for pathologic analysis.

Lee et al. performed a nonrandomized, retrospective study reviewing the incidence, severity and risk factors of BNC in patients treated with TURP alone and TURP plus transurethral incision (TUI) of the bladder neck - a prophylactic treatment for BNC - to test the validity of TURP plus TUI as an alternative to TUIP. A total of 1,135 patients were reviewed: 667 treated with TURP and 468 treated with TURP plus TUI. Median followup was 37.9 months.

The incidence of BNC with TURP and TURP plus TUI was $12.3 \%$ and $6 \%$, respectively. In patients with an adenoma weight of $>30 \mathrm{~g}$, TURP plus TUI completely prevented the incidence of BNC. Multivariate analysis revealed that adenoma weight, surgical type and absence of vesical stones were significant risk factors for BNC, although personal diathesis may play a role.

In summary, TURP plus TUI can be used as an alternative to TUIP in selected patients. The authors suggest that further work be carried out to determine the exact mechanism of BNC.

Original article Lee YH et al. (2005) Comprehensive study of bladder neck contracture after transurethral resection of prostate. Urology 65: 498-503

\section{Immunotherapy reduces incidence of urinary tract infections in women}

A new study confirms that the immunotherapeutic agent OM-89 reduces recurrent urinary tract infection (UTI). OM-89 is a lyophilized extract of selected Escherichia coli strains in a capsule formation, containing $6 \mathrm{mg}$ of bacterial extract. Previous clinical trials lasting up to 6 months have shown that OM-89 decreases episodes of UTI in adult, pediatric, pregnant, postmenopausal or paraplegic patients compared with placebo.

Bauer and co-workers carried out a multicenter, double-blind study to investigate the long-term preventive effects of OM-89 in a large cohort of exclusively female patients. A total of 454 patients with recurrent UTI were enrolled in the study, with 232 randomized to OM-89 and 222 to placebo. The dosage regimen was 1 capsule daily during months 1-3 and 1 capsule daily for the first 10 days of months 7-9.

Multiple regression analysis showed that OM-89 significantly reduced the incidence of UTI, by $34 \%$ compared with placebo, over a 12 month study period. The incidence of UTI was $43 \%$ lower compared with placebo between month 7 and the end of the study, indicating that booster courses of treatment could be beneficial. OM-89 was well tolerated and had a good safety profile.

Original article Bauer HW et al. (2005) A long-term, multicenter, double-blind study of an Escherichia coli extract (OM-89) in female patients with recurrent urinary tract infections. Eur Urol 47: 542-548

\section{Protein profiling of urine in the diagnosis of bladder cancer}

Protein profiling of urine samples can be used to detect and diagnose transitional-cell carcinoma of the bladder with high sensitivity and specificity, according to a recent study by Liu et al.

The team used a technique called SURFACEENHANCED LASER DESORPTION/IONIZATION TIME-OFFLIGHT MASS SPECTROMETRY (SELDI-TOF-MS) COUpled with a tree analysis pattern. Altogether, 172 specimens were included in the study. These were obtained from patients with transitionalcell carcinoma $(n=83)$, patients with other urogenital diseases $(n=36)$, and healthy controls $(n=53)$. A data set of 104 "training samples" was used to generate the tree analysis pattern. This was then tested using a separate data set of 68 "test samples".

Overall, this approach correctly classified approximately $93 \%$ of the training samples and $72 \%$ of the test samples. The authors note that the sensitivity was higher for samples taken from grade III carcinomas (95.2\%) than from grades I and II (79.2\%), suggesting that this approach is better suited to the detection of high-grade cancers.

SELDI protein profiling of urine samples is non-invasive, unlike cytoscopic examination and bladder biopsy, and does not have the high rate of false negatives associated with urine cytology. Added advantages are its speed, high-throughput capability, and sensitivity. In conclusion, the authors suggest

\section{GLOSSARY}

SURFACE-ENHANCED LASER DESORPTION/ IONIZATION TIMEOF-FLIGHT MASS SPECTROMETRY (SELDITOF-MS)

Method for sorting the proteins in a biologic sample into groups based on common properties, visualised as peaks, read by a ProteinChip ${ }^{\circledR}$ Reader Proteins of interest can be purified 